Hautarzt 2011 • 62:362-367

DOI 10.1007/s00105-010-2084-7

Online publiziert: 7. April 2011

(c) Springer-Verlag 2011

\author{
M. Möhrle ${ }^{1,2} \cdot$ V. Lichte $^{1} \cdot$ H. Breuninger ${ }^{1}$ \\ ${ }^{1}$ Universitäts-Hautklinik Tübingen \\ 2 Praxisklinik Tübingen - Haut und Venen, Dr. med. Arno Bern \\ und Prof. Dr. med. Matthias Möhrle, Tübingen
}

\title{
Operative Therapie von akral lokalisierten Melanomen
}

Die lückenlose histologische Schnittrandkontrolle wurde von Frederic E. Mohs begründet [16]. Daher wird die mikrographische Chirurgie oder mikroskopisch kontrollierte Chirurgie (MKC) im englischen Sprachraum meist als „Mohs' Surgery“ bezeichnet. Die mikroskopisch kontrollierte Chirurgie stellt nicht eine chirurgische Prozedur, sondern eher eine histopathologische Technik dar [13]. Aus diesem Grund wurde die Bezeichnung „dreidimensionale (3D-)Histologie“ eingeführt $[20,22] .3 \mathrm{D}$-Histologie bedeutet die vollständige histologische Untersuchung der $3 \mathrm{D}$-Ränder von Tumorexlung. zidaten. Die Technik eignet sich für Tumoren mit einem kontinuierlichen Ausbreitungsverhalten [3]. Das ALM, das an Händen und Füßen lokalisiert ist, breitet sich ähnlich wie das Lentigo-maligna-Melanom in sonnenlichtexponierter Haut zunächst über kontinuierlich wachsende Tumorstränge aus und geht erst nach Monaten bzw. Jahren in ein vertikales invasives Wachstumsmuster über [ 5,7 , 15, 25]. Die 3D-Histologie ermöglicht im Gegensatz zur konventionellen Histologie („Brotlaibtechnik“) eine lückenlose Kontrolle des horizontalen Tumorwachstums mit reduzierten Sicherheitsabständen und kann den Patienten ggf. Amputationen ersparen. Unter Einsatz der 3D-Histologie werden akral lokalisierte Melanome lokal chirurgisch mit einem guten funktionellen und kosmetischen Ergebnis behandelt.

Ein etabliertes Verfahren der $3 \mathrm{D}$-Histologie bei akral lokalisierten Melanomen ist die modifizierte „Tübinger Torte“ ohne histologische Kontrolle der Tumorbasis (• Abb. 1).

\section{Konventionelle Histologie vs. 3D-Histologie beim akrolentiginösen Melanom}

Seit der Beschreibung von Reed im Jahre 1976 „ALM is a very aggressive, malignant cutaneous tumour wihich a predilection for the plantar surface of the feet, palm of the hand, and the digits... These lesions have a propensity to metastasize to the central nervous system, liver, lungs, bone, and lymph nodes. Due to the aggressi-
Abb. $1 \Delta$ Tübinger Torte. Der sichtbare Tumor (schwarz) wird mit einem kleinen Sicherheitsabstand mit senkrechten (oder evertierenden) Rändern exzidiert. Eine Fadenmarkierung wird bei $12.00 \mathrm{Uhr}$ zur topographischen Orientierung angebracht. Im Bereich von 5.00-7.00 Uhr findet sich ein subklinischer Tumorausläufer (oben). Der Rand wird abpräpariert (Mitte), in einer Histologiekassette plan fixiert, in Paraffin eingebettet und danach vertikal geschnitten (rechts). Der subklinische Tumorausläufer wird richtig positiv diagnostiziert, da der Schnittrand vollständig beurteilt werden kann. (Aus [21]) 
ve nature of these lesions, early recognitions and treatment, consisting of excision in toto with clear margins, are imperative" besteht kein Konsens über die diagnostischen, histologischen und klinischen Kriterien des ALM.

Zahlreiche Arbeiten zum ALM besitzen geringe Fallzahlen von Patienten unterschiedlicher ethnischer Herkunft (Übersicht in [11]).

Zur histologischen Schnittrandkontrolle beim ALM wurde kürzlich die sog. „Spaghetti-Technik“ publiziert [34]: Zunächst wurde lediglich der Sicherheitsabstand exzidiert, während der Tumor in situ belassen wurde. Nach der histologischen Untersuchung der äußeren Schnittränder erfolgte bei positiven Rändern eine topographisch orientierte Nachexzision. Bei tumornegativen Schnitträndern wurde der noch verbleibende Tumor reseziert und der Defekt plastisch-rekonstruktiv verschlossen. Das Vorgehen bei der "Spaghetti-Technik“ entspricht daher genau der „Wallgraben-Technik“, die für bestimmte Tumore des Gesichtsbereichs empfohlen wurde $[2,12]$. Wird eine Sekundärheilung beim ALM angestrebt (- Abb. 2) bedeutet die „Spaghetti-Technik“ immer eine zusätzliche Operation der Tumorresektion. Sie bringt dadurch keinen Vorteil. Darüber hinaus erscheint es problematisch, einen malignen Tumor als „Platzhalter“ bis zum Vorliegen des Ergebnisses der histologischen Schnittränder in situ zu belassen.

Kürzlich wurde die bislang größte Serie von ALM-Patienten publiziert [11]:

Im Zeitraum von 1980-2006 wurden an der Universitäts-Hautklinik Tübingen 244 Patienten mit einem ALM im klinischen Stadium I/II (44\% Männer, 56\% Frauen) operiert. Bei 133 Patienten (55\%) wurde das Gewebe mittels 3D-Histologie untersucht, bei $45 \%$ mittels konventioneller Histologie. Risikofaktoren für das rezidivfreie Überleben und das Gesamtüberleben wurden univariat und multivariat evaluiert. Das mediane Alter der Patienten betrug 63 Jahre (26-87 Jahre), die mediane Tumordicke $2,0 \mathrm{~mm}$. In der multivariaten Analyse konnten Ulzeration, konventionelle Histologie und die Tumordicke als unabhängige Risikofaktoren für das rezidivfreie Überleben und Gesamtüberleben identifiziert werden [11].

Hautarzt 2011 · 62:362-367 DOI 10.1007/s00105-010-2084-7

(c) Springer-Verlag 2011

\section{Möhrle $\cdot$ V. Lichte $\cdot H$. Breuninger Operative Therapie von akral lokalisierten Melanomen}

Zusammenfassung

Akrolentiginöse Melanome (ALM) umfassen 4-10\% der kutanen Melanome bei Hellhäutigen. Patienten mit ALM wird oft eine schlechtere Prognose zugeschrieben, meist aufgrund zu später Diagnosestellung. Unter Einsatz der 3D-Histologie können akral lokalisierte Melanome mit kontinuierlichem Ausbreitungsmuster lokal chirurgisch mit kleineren Sicherheitsabständen und einem guten funktionellen und kosmetischen Ergebnis behandelt werden. In einer Studie wurden bei $244 \mathrm{~Pa}$ tienten mit ALM die konventionelle Histologie vs. 3D-Histologie neben anderen prognostischen Parametern verglichen. Klinische und chirurgische Risikofaktoren beeinflussen die Prognose des ALM. Tumordicke und Ulzeration sind die wichtigsten Risikofaktoren. Die 3D-Histologie in Paraffintechnik ermöglicht es, Sicherheitsabstände zu reduzieren und Lokalrezidive zu vermeiden. Subunguale Melanome machen nur etwa $2-3 \%$ der kutanen Melanome beim kaukasischen und etwa 20\% der Melanome beim afrikanischen oder asiatischen Hauttyp aus und werden klinisch häufig fehldiagnostiziert. Sie sind oft an Daumen und Großzehe lokalisiert. Die Entfernung von subungualen Melanomen mit 3DHistologie und tumorfreien Schnitträndern unter Einschluss der Nagelmatrix kann als sichere Strategie angesehen werden, welche die Prognose nicht beeinträchtigt. Funktion und Kosmetik eines Fingers oder Zehs bleiben erhalten. Amputationen bei subungualen Melanomen sollten fortgeschrittenen Verläufen mit Knochen- oder Gelenkbefall vorbehalten bleiben.

\section{Schlüsselwörter}

Akrolentiginöses Melanom · Melanom . Mikroskopisch kontrollierte Chirurgie . 3D-Histologie · Subunguales Melanom

\section{Operative therapy of acral melanomas}

\section{Abstract}

Acral lentiginous melanomas (ALM) represent $4-10 \%$ of cutaneous melanomas in white populations. Patients with ALM seem to have a poor prognosis, often due to late diagnosis. Micrographic surgery (3D-histology) is not seen as surgical procedure but more as histopathological technique. With micrographic surgery, continuously spreading ALM can be excised with smaller excision margins and good functional and cosmetic outcomes. In a recent study, 244 patients with ALM were compared using conventional histology versus 3D-histology. Clinical and surgical risk factors influence the prognosis of ALM. Tumor thickness and ulceration are the most important prognostic factors. 3D-histology with paraffin technique (optionally combined with immunohistological methods) can reduce excision margins and avoid local recurrences. Subungual melanomas represent only 2-3\% of cutaneous melanomas in Caucasian and $20 \%$ in African or Asian skin type and are often clinically misdiagnosed. They are often localized on the thumb or great toe, which are most important for the function of the affected limb. The excision of subungual melanoma with 3D-histology and tumor-free excision margins including the nail matrix can be seen as a safe surgical strategy, which does not hazard the prognosis of the patient. Function and cosmesis of the finger or toe are preserved. Amputation in subungual melanoma is not recommended and should be reserved only for infiltrating melanomas with affection of the bone or joint.

\section{Keywords}

Acral lentiginous melanoma - Melanoma .

Micrographic surgery · 3D-histology .

Subungual melanoma 


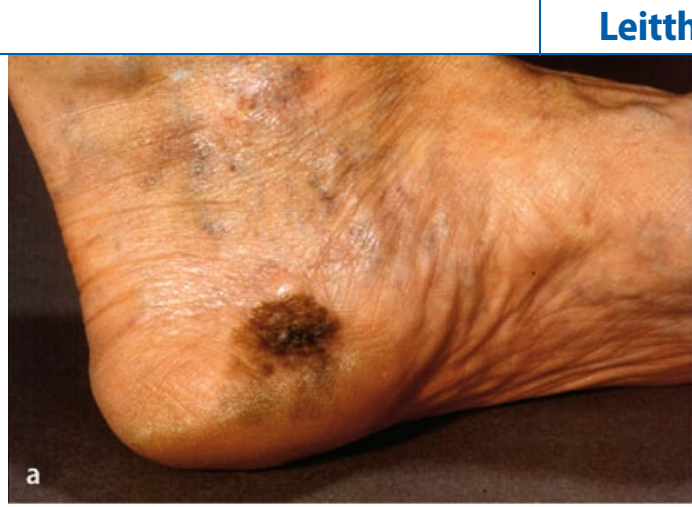

Leitthema
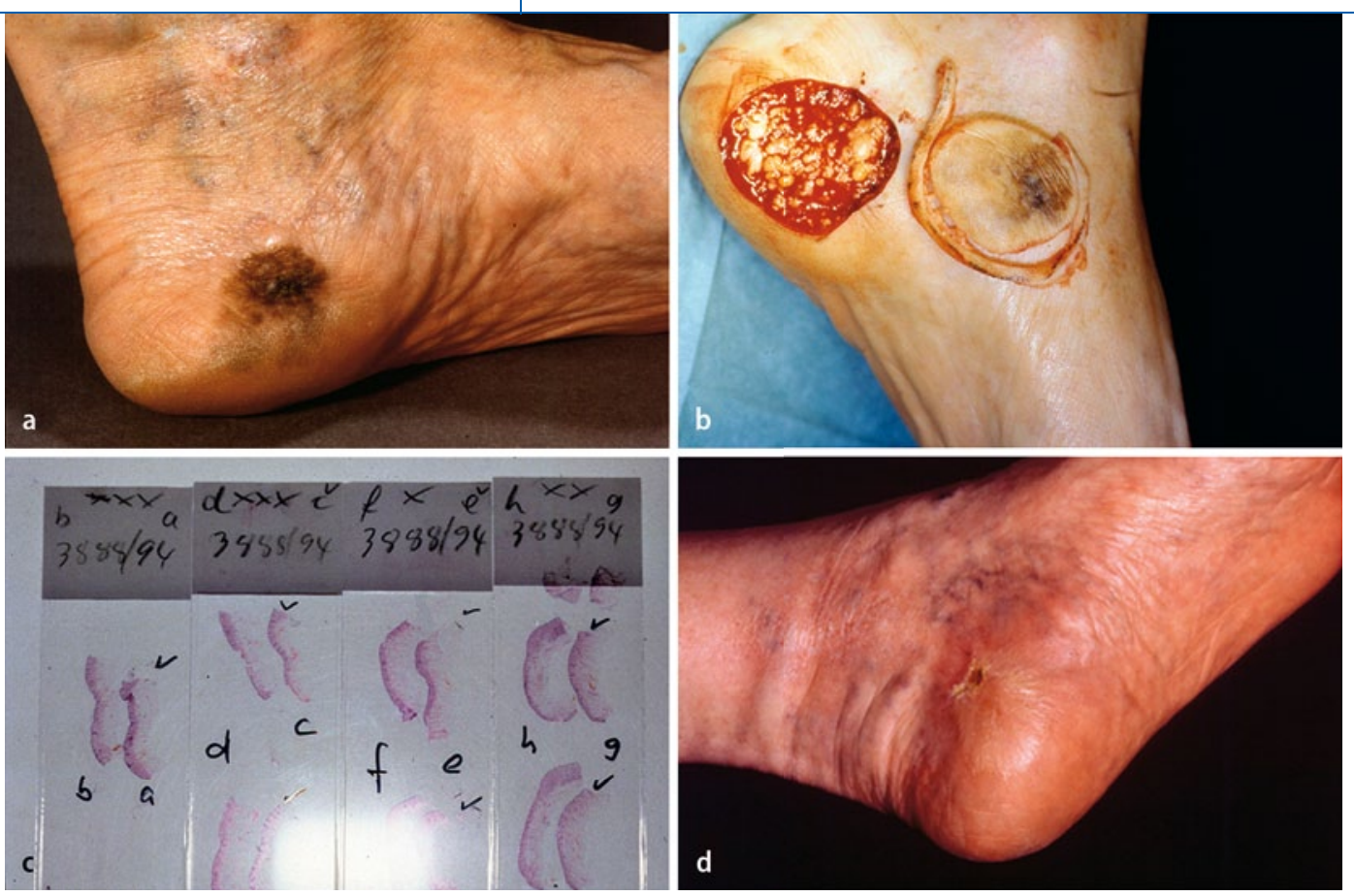

Abb. $2 \triangleleft$ Exzision eines akrolentiginösen Mela-

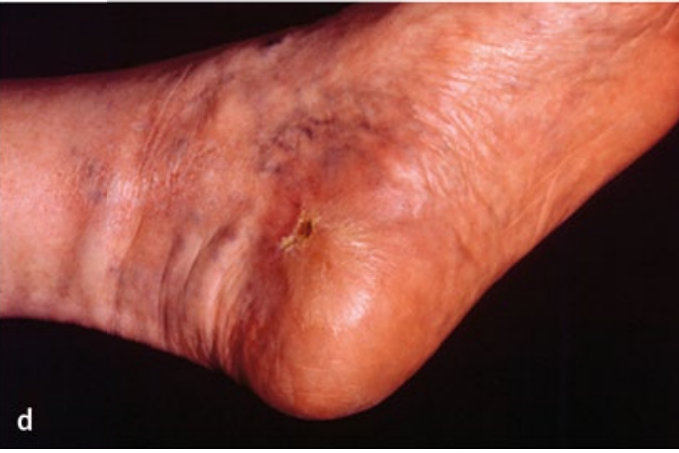
noms (ALM) mit 3D-Histologie. a Patient mit einem ALM an der linken Ferse. b Exzision mit $5 \mathrm{~mm} \mathrm{Si-}$ cherheitsabstand. Präparation der Ränder für die 3D-Histologie. c Alphabetisch orientierte histologische Schnitte. d Ergebnis nach 6 Wochen Sekundärheilung. (Aus [11], mit freundl. Genehmigung Wiley-Blackwell)

In einer relativ großen Serie mit 126 ALM-Patienten der Universität Lyon waren die Tumordicke, männliches Geschlecht und ein amelanotischer Tumor Risikofaktor für das Gesamtüberleben. Darüber hinaus stellten sich mitotische Aktivität, Mikrosatelliten und Invasionslevel als Risikofaktoren für das rezidivfreie Überleben dar [26].

\section{( ) Unter Einsatz der 3D-Histologie waren die Sicherheitsabstände kleiner, ohne das Risiko für ein Lokalrezidiv zu erhöhen}

In der Tübinger ALM-Studie wurde darüber hinaus der Einfluss des Sicherheitsabstandes und der histologischen Schnittrandkontrolle auf die Prognose untersucht [11]. Unter Einsatz der 3D-Histologie waren die Sicherheitsabstände kleiner ( $\mathrm{p}=0,0003$; 7 vs. $20 \mathrm{~mm}$ ), ohne das Risiko für ein Lokalrezidiv zu erhöhen. Patienten, die mit $3 \mathrm{D}$-Histologie operiert wurden, hatten eine 5-Jahres-Überlebensrate von $81 \%$, Patienten, die mit konventioneller Histologie operiert wurden, von $63 \%$. Die Zahl der echten Lokalrezidive betrug 9\% bei reduziertem Sicherheitsabstand und $3 \mathrm{D}$-Histologie vs. $10 \%$ bei weitem Sicherheitsabstand und konventioneller Histologie. Lokalrezidive waren ohne statistischen Einfluss auf das Gesamtüberleben. Trotzdem erscheint es wichtig, Lo- kalrezidive frühzeitig zu diagnostizieren und operativ zu entfernen. Regelmäßige Nachsorgeuntersuchungen sind beim ALM auch nach $3{ }_{3} \mathrm{D}$-Histologie indiziert.

Die Autoren erwarteten jedoch eine geringere Rate von Lokalrezidiven nach ${ }_{3} \mathrm{D}$-Histologie. Es stellte sich daher die Frage, ob das Konzept der 3D-Histologie beim ALM falsch ist und man zu großen Sicherheitsabständen mit konventioneller Histologie zurückkehren sollte oder die ${ }_{3} \mathrm{D}$-Prozedur verbessert werden könnte: Um etwaige diagnostische Lücken bei der $3 \mathrm{D}$-Histologie aufzudecken, wurden retrospektiv die Präparate von 10 Patienten untersucht, die bei „negativer“ 3 D-Histologie doch ein Lokalrezidiv entwickelten - wobei 5 Lokalrezidive lediglich In-situRezidive darstellten. Bei dieser Überprüfung zeigte sich, dass die initial falsch-negativen Präparate eine schlechte Schnittqualität aufwiesen $(n=6)$. In der retrospektiven Untersuchung fanden sich isolierte atypische Zellen $(n=4)$ oder gruppierte atypische Zellen $(\mathrm{n}=1)$. Unter $\mathrm{Zu}$ hilfenahme der Immunhistologie (AntiMelan-A) wurden 4 der Randschnitte eindeutig positiv und 5 Präparate als unklar mit atypischen Einzelzellen beurteilt. Der Einsatz der Immunhistologie (AntiMelan-A) könnte daher die Sicherheit der ${ }_{3} \mathrm{D}$-Histologie beim ALM steigern und sollte in Erwägung gezogen werden [11].
Es gibt nur wenige Untersuchungen zur Immunhistochemie beim ALM. Am sensitivsten in der Paraffinhistologie gelten Anti-Protein-S1oo-Antikörper (95\%), gefolgt von Anti-HMB45-Antikörpern (8o\%) und Anti-Mart1/Melan-A-Antikörpern $(79 \% ;[26,31])$.

Die aktuellen Leitlinien empfehlen die Exzision des ALM mit reduziertem Sicherheitsabstand und $3 \mathrm{D}$-Histologie $[6$, $10,12]$.

\section{Chirurgie und 3D-Histologie bei subungualen Melanomen}

Oft besteht eine große Zurückhaltung in der histologischen Abklärung von pigmentierten subungualen Läsionen. Aus dermatochirurgischer und dermatopathologischer Sicht stellt die Shave-Biopsie/Shave-Exzision von frühen Pigmentmalen des Nagelbetts eine Methode der Wahl dar. In den meisten Fällen sind die Histologie und das kosmetische Ergebnis nach Abheilung der Biopsie/Exzision hervorragend. Die Shave-Exzision von Pigmentläsionen der Nagelmatrix ist daher nach der Dermoskopie als minimal-invasive Diagnostik früh in Erwägung zu ziehen $[9,29]$.

Subunguale Melanome sind sehr selten und machen nur etwa $2-3 \%$ der kutanen Melanome beim kaukasischen $[1,19]$ und etwa $20 \%$ der Melanome beim afrika- 
nischen [24] oder asiatischen [32] Hauttyp aus. Daumen und Großzehe, also die für Greiffunktion und Gang funktionell bedeutsamsten Phalangen, sind überproportional häufig von subungualen Melanomen betroffen [18]. Aufgrund von klinischen Fehldiagnosen wird die histopathologische Diagnose subunguales Melanom oft verspätet gestellt [14].

Hutchinson beschrieb im Jahr 1886 erstmals das subunguale Melanom und empfahl ein radikales chirurgisches Vorgehen („early amputation is demanded“). Seither wird beim subungualen Melanom radikal operiert. Amputationen wurden im Niveau der Metakarpal-/Metatarsalknochen [24] oder im Metakarpal-/Metatarsalgelenk $[12,30,33]$ postuliert. Manche Autoren empfahlen, für ein physiologisches Gangbild das 1. Metatarsalköpfchen zu belassen [30, 33]. Allgemein gilt: Je länger der verbliebene Großzehenanteil, desto besser die physiologische Abrollbewegung.

Der Verlust eines Fingers und hier insbesondere des Daumens führt zu einer deutlichen Funktionseinbuße und schlechter Kosmetik. Daher wurden reduzierte Amputationen empfohlen mit dem Ziel, bei ausreichenden Sicherheitsabständen Finger möglichst lange zu erhalten [30]. Am Daumen sollte proximal des Interphalangealgelenks, an den anderen Fingern proximal des distalen Interphalangealgelenks abgesetzt werden [30, 33]. Später wurden Amputationen im distalen Interphalangealgelenk diskutiert [4, 23]. Diese traditionelle chirurgische Therapie ist hinsichtlich ihres Nutzens auf Rezidivfreiheit und Überleben nicht belegt.

Über den Einsatz der 3 D-Histologie bei subungualem Melanom in situ wurde anekdotisch berichtet.

In einer aktuellen Arbeit wurde die prognostische Bedeutung von klinischen Risikofaktoren, dem Amputationslevel und der $3 \mathrm{D}$-Histologie beim subungualen Melanom untersucht [19]. Unter 3960 Patienten mit einem Melanom im Stadium I/II fanden sich 62 Patienten mit einem subungualen Melanom; 31 Patienten erhielten eine Amputation im oder proximal des distalen Interphalangealgelenks, 31 Patienten wurden "funktionell“ operiert mit einer lokalen Exzision des Tumors mit kleinem Sicherheitsab- 


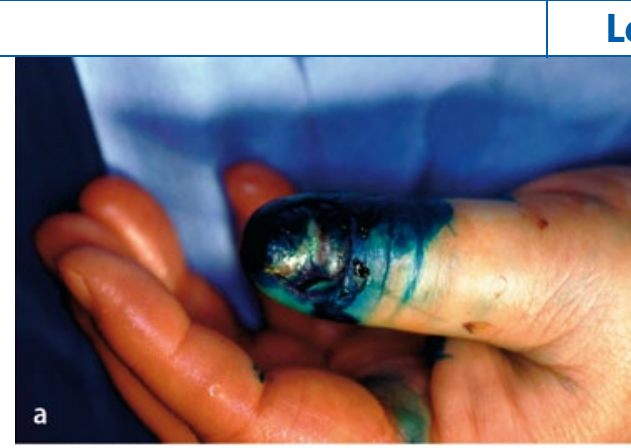

\section{Leitthema}
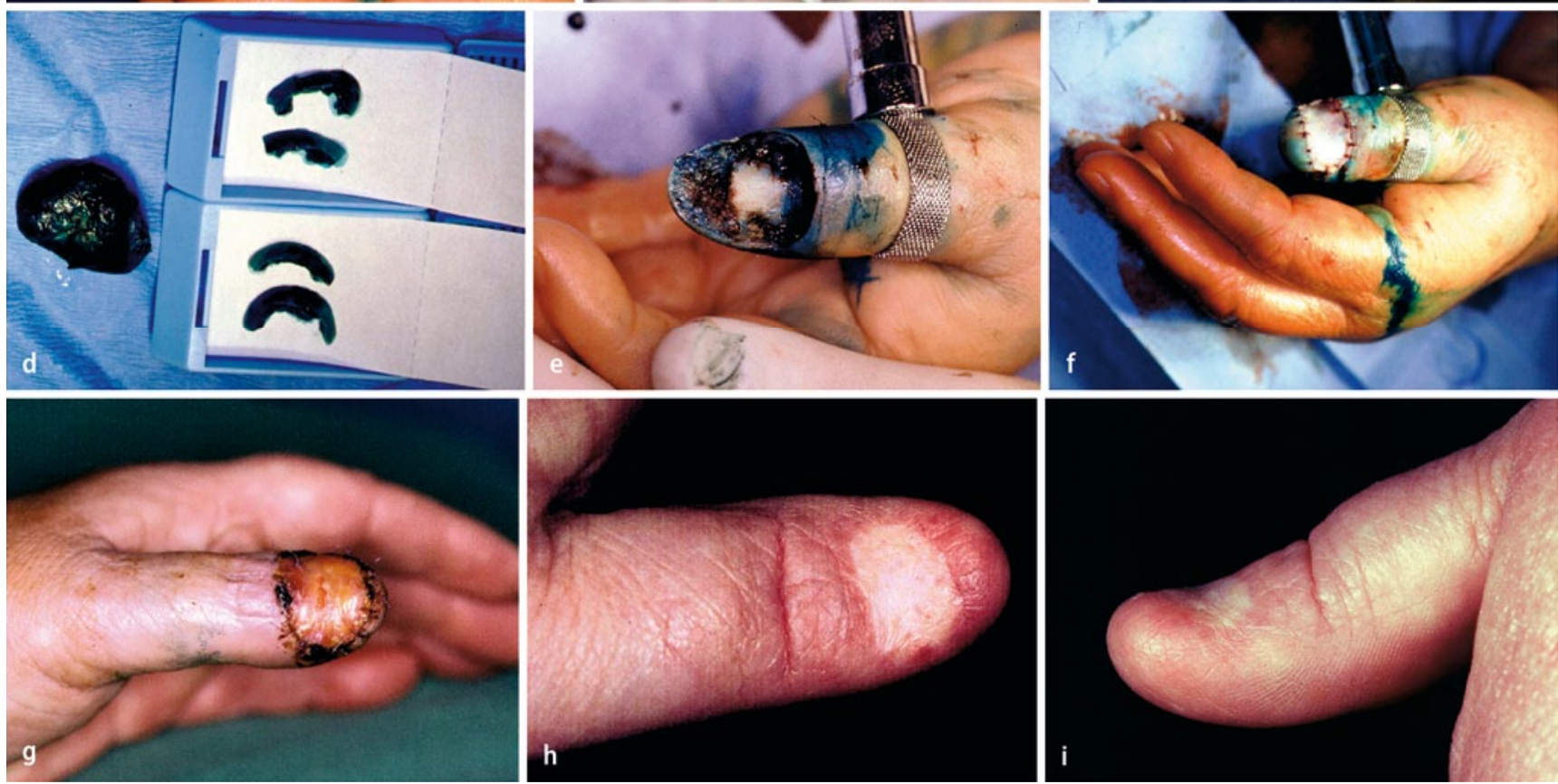

Abb. $3 \Delta$ Subunguales Melanom (Tumordicke 3,25 mm, Level V) am linken Daumen einer 54-jährigen Patientin. a Präoperativ. $\mathbf{b}$ Exzision des Tumors einschließlich des gesamten Nagelapparates bis zum Knochen der Endphalanx mit einem Sicherheitsabstand von $5 \mathrm{~mm}$. c Präparation des Randes vom Exzisat in 4 Teilstücken (0.00-3.00 Uhr, 3.00-6.00 Uhr, 6.00-9.00 Uhr, 9.00-12.00 Uhr). d Histologische Einbettung der Randstücke. e Resektion des Processus unguinatus. f Defektdeckung mit einem Vollhauttransplantat. $\mathbf{g}$ Klinischer Befund 1 Woche postoperativ. $\mathbf{h}$, i Hervorragendes funktionelles und kosmetisches Ergebnis 6 Monate postoperativ. (Aus [21])

stand und, wenn notwendig, nur partieller Endgliedresektion (Processus unguinatus). Mit Skalpell und Schere wurden en bloc der klinisch sichtbare Tumor, Nagelbett und Matrixhörner mit einem initialen Sicherheitsabstand von $5 \mathrm{~mm}$ entfernt. Der Defekt wurde dann durch die Kombination einer modifizierten Fischmaulplastik von volar und einer Vollhautplastik gedeckt. Die Operation lässt sich in örtlicher Betäubung mit einer Blutsperre durchführen (- Abb. 3). Es wurde, wie in - Abb. 1 beschrieben, eine 3D-Histologie durchgeführt.

\section{- Nach „funktioneller“ Operation oder} unter Einsatz der 3D-Histologie war das rezidivfreie Überleben nicht schlechter als nach einer Amputation.
Es fanden sich nach „funktioneller“ Operation jedoch 2 echte Lokalrezidive (3,2\%). Diese Lokalrezidive sind nicht als Versagen des operativen und histologischen Prinzips zu werten. In einem Fall wurde lediglich eine Exzision mit Sicherheitsabstand ohne $3 \mathrm{D}$-Histologie durchgeführt, im anderen Fall waren die histologischen Randschnitte artifiziell verändert und wurden fälschlicherweise als tumorfrei befundet.

Die Größe des Sicherheitsabstandes war ohne Bedeutung für das Gesamtüberleben oder das rezidivfreie Überleben.

In der multivariaten Analyse stellten sich die Tumordicke (RR 1,003 pro 1/100 $\mathrm{mm}$ ) und die Durchführung einer Amputation (im Gegensatz zur „funktionellen Chirurgie“; RR 5,52) als unabhän- gige Risikofaktoren für das rezidivfreie Überleben dar.

\section{Fazit für die Praxis}

- Klinische und chirurgische Risikofaktoren beeinflussen die Prognose des ALM.

- Tumordicke und Ulzeration sind die wichtigsten Risikofaktoren.

- Die 3D-Histologie in Paraffintechnik (ggf. mit Immunhistologie) ermöglicht es, Sicherheitsabstände zu reduzieren und Lokalrezidive zu vermeiden.

- Immunhistologie verbessert die Genauigkeit der Schnittrandkontrolle.

- Die Entfernung von subungualen Melanomen mit 3D-Histologie und tumorfreien Schnitträndern unter Ein- 
schluss der Nagelmatrix kann als sichere Strategie angesehen werden, welche die Prognose nicht beeinträchtigt.

- Funktion und Kosmetik eines Fingers oder Zehs bleiben erhalten.

- Amputationen bei subungualen Melanomen können nicht mehr generell empfohlen werden und sollten fortgeschrittenen Verläufen mit Knochenoder Gelenkbefall vorbehalten bleiben.

\section{Korrespondenzadresse \\ Prof. Dr. M. Möhrle}

Praxisklinik Tübingen - Haut und Venen,

Dr. med. Arno Bern und

Prof. Dr. med. Matthias Möhrle

Europaplatz 2, 72072 Tübingen

matthias.moehrle@med.uni-tuebingen.de

Interessenkonflikt. Der korrespondierende Autor gibt an, dass kein Interessenkonflikt besteht.

\section{Literatur}

1. Blessing K, Kernohan NM, Park KG (1991) Subungual malignant melanoma: clinicopathological features of 100 cases. Histopathology 19:425-429

2. Blum A, Möhrle M (2004) Wallgrabentechnik. Zweizeitige operative Versorgung von ausgedehnten Basalzellkarzinomen im Lippenbereich. Hautarzt 55:869-873

3. Breuninger $H$, Schlagenhauff B, Stroebel W et al (1999) Patterns of local horizontal spread of melanomas: consequences for surgery and histopathologic investigation. Am J Surg Pathol 23:14931498

4. Brochez L, Verhaeghe E, Sales F et al (2000) Current guidelines in melanoma treatment. Melanoma Working Group of Gent and Bordet. Dermatology 200:160-166

5. Coleman WP, Loria PR, Reed RJ, Krementz ET (1980) Acral lentiginous melanoma. Arch Dermatol 116:773-776

6. Garbe C, Schadendorf D, Stolz W et al (2008) Short German guidelines: malignant melanoma. J Dtsch Dermatol Ges 6(Suppl 1):S9-S14

7. Harmelin ES, Holcombe RN, Goggin JP et al (1998) Acral lentiginous melanoma. J Foot Ankle Surg 37:540-545

8. Hutchinson J (1886) Melanosis often not black: melanotic whitlow. Br Med J 1:491

9. Jellinek N (2007) Nail matrix biopsy of longitudinal melanonychia: diagnostic algorithm including the matrix shave biopsy. J Am Acad Dermatol 56:803810

10. Leiter U, Eigentler TK, Forschner A et al (2010) Excision guidelines and follow-up strategies in cutaneous melanoma: facts and controversies. Clin Dermatol 28:311-315

11. Lichte V, Breuninger $H$, Metzler $G$ et al (2009) Acral lentiginous melanoma: conventional histology vs. three-dimensional histology. Br J Dermatol 160:591-599
12. Lingam MK, McKay AJ, Mackie RM, Aitchison T (1995) Single-centre prospective study of isolated limb perfusion with melphalan in the treatment of subungual malignant melanoma. Br J Surg 82:1343-1345

13. Löser C, Rompel R, Breuninger H et al (2010) Microscopically controlled surgery (MCS). J Dtsch Dermatol Ges 8:920-925

14. Metzger S, Ellwanger U, Stroebel W et al (1998) Extent and consequences of physician delay in the diagnosis of acral melanoma. Melanoma Res 8:181-186

15. Michaelsen C, Breuninger $H$, Rassner G, Dietz K (1990) Der subklinische Anteil im Randbereich der Lentigo maligna und des Lentigo-maligna-Melanoms. Hautarzt 41:142-145

16. Mohs FE (1941) Chemosurgery: a microscopically controlled method of cancer excision. Arch Surg 42:279-281

17. Möhrle M (2003) Mikrographisch kontrollierte Chirurgie (3D-Histologie) beim Melanom. JDDG a 1:869-875

18. Möhrle M, Häfner HM (2002) Is subungual melanoma related to trauma? Dermatology 204:259-261

19. Möhrle M, Metzger S, Schippert W et al (2003) "Functional" surgery in subungual melanoma. Dermatol Surg 29:366-374

20. Möhrle M, Kraemer A, Schippert W et al (2004) Clinical risk factors and prognostic significance of local recurrence in cutaneous melanoma. $\mathrm{Br} J \mathrm{Der}-$ matol 151:397-406

21. Möhrle M, Breuninger H (2006) Chirurgie des Melanoms in akraler und fazialer Lokalisation mit 3DHistologie (mikrographische/histographische Chirurgie). In: Garbe C (Hrsg) Management des Melanoms. Springer Medizin, Heidelberg, S 207-214

22. Möhrle M, Breuninger $H$, Röcken M (2007) A confusing world: what to call histology of three-dimensional tumour margins? J Eur Acad Dermatol Venereol 21:591-595

23. O'Leary JA, Berend KR, Johnson JL et al (2000) Subungual melanoma. A review of 93 cases with identification of prognostic variables. Clin Orthop Relat Res 378:206-212
24. Pack GT, Oropeza R (1967) Subungual melanoma. Surg Gynecol Obstet 124:571-582

25. Paladugu RR, Winberg CD, Yonemoto RH (1983) Acral lentiginous melanoma. A clinicopathological study of 36 patients. Cancer 521:161-168

26. Phan A, Touzet S, Dalle S et al (2006) Acral lentiginous melanoma: a clinicoprognostic study of 126 cases. Br J Dermatol 155:561-569

27. Phan A, Touzet S, Dalle S et al (2007) Acral lentiginous melanoma: histopathological prognostic features of 121 cases. Br J Dermatol 157:311-318

28. Reed RJ (1976) Acral lentiginous melanoma. In: Hartman W, Kay S, Reed RJ (Hrsg) New concepts in surgical pathology of the skin. Wiley, New York, $S$ $89-90$

29. Richert B, Lateur N, Theunis A, Andre J (2009) New tools in nail disorders. Semin Cutan Med Surg 28:44-48

30. Ross MI, Balch CM (1998) Surgical treatment of primary melanoma. In: Balch CM, Houghton AN, Sober AJ (Hrsg) Cutaneous melanoma, 3. Aufl. Quality Medical Publishing, St. Louis, Missouri, S 141153

31. Stalkup JR, Orengo IF, Katta R (2002) Controversies in acral lentiginous melanoma. Dermatol Surg 28:1051-1059

32. Takematsu H, Obata M, Tomita Y et al (1985) Subungual melanoma. A clinicopathologic study of 16 Japanese cases. Cancer 55:2725-2731

33. Wagner JD, Gordon MS, Chuang TY, Coleman JJ (2000) Current therapy of cutaneous melanoma. Plast Reconstr Surg 105:1774-1799

34. Gaudy-Marqueste C, Perchenet AS, Taséi AM et al. (2011) The "spaghetti technique", an alternative to Mohs surgery or staged surgery for problematic lentiginous melanoma (lentigo maligna and acral lentiginous melanoma). J Am Adac Dermatol 64:113-118

\section{www.SpringerMedizin.de/dermatologie}

\section{Alles rund um Ihr Fachgebiet}

Wählen Sie ab sofort den direkten Weg zu News

und Fachbeiträgen aus Ihrem Fachgebiet:

Auf www.SpringerMedizin.de/dermatologie

halten wir Sie immer auf dem Laufenden.

Hier erwartet Sie ein umfassendes Informationsangebot:

- News aus Berufs- und Gesundheitspolitik

- Ausgewählte Meldungen und Beiträge aus Ihrem Fachgebiet

- Die wichtigsten Studien und Publikationen - für Sie zusammengefasst und kommentiert

- Kompetente Unterstützung bei schwierigen Fragestellungen durch unsere Expertenräte

- Medizinische Fortbildung in Form von Bilderstrecken, Podcasts bis hin zu Reviews und

CME-Beiträgen aus den Fachzeitschriften von Springer Medizin

So einfach geht's: Nach einmaliger Registrierung steht Ihnen ein umfangreiches kostenloses Angebot zur Verfügung.

SpringerMedizin.de - Das Internet der Ärzte 